\title{
Privacy Concern and Trust in Using Social Network Sites: A Comparison between French and Chinese Users
}

\author{
Li Chen and Ho Keung Tsoi \\ Department of Computer Science, Hong Kong Baptist University \\ Hong Kong, China \\ \{lichen, hkt.soi\} @comp.hkbu.edu.hk
}

\begin{abstract}
Though privacy and trust have been studied in the social network site (SNS), few have identified the relationships among users' privacy concern, trust and their actual usage behavior in SNS. Moreover, little attention has been paid to investigating the differences between users from different cultural contexts. In this paper, we have been engaged in addressing these concerns by surveying two typical user groups. The analysis of their answers showed that French and Chinese are not only significantly different regarding privacy and trust belief in SNS, but also act significantly different in disclosing personal information, posting messages, and developing new relationships. Furthermore, the effect of privacy concern and trust on users' visiting frequency and willingness to meet new people is also found different between the two groups.
\end{abstract}

Keywords: social network site, privacy concern, trust, usage behavior, cultural differences.

\section{Introduction}

With the increasing popularity of social network sites (SNS) worldwide, more and more attentions have been paid to understanding users' behavior when they are using the sites. One major concern is whether users are willing to disclose their personal info and tend to meet new people through the SNS. It is also interesting to know whether users actively post information regularly and whom they allow to access the info. These issues are in nature related to users' privacy concern and trust formation in the site. Indeed, trust has long been recognized as the primary factor leading to successful online transactions and interaction. Prior trust-related investigations in ecommerce sites showed that higher trust can prompt users to have more intention to purchase a product, and to return to the site for repeated uses [3]. On the other hand, it has been recognized that if a user worries his/her personal info will be used by the site for other purchases, s/he will unlikely trust the site and hence not be so active in disclosing her/his personal info. Thus, privacy concern seems to be a dominant factor in influencing users' trust building, and trust would further be the antecedent affecting users' actual behavior in the site.

However, though the concept "trust" has been extensively studied in SNS, the focus has been mainly on testing and enhancing trust relation between users [2], not 
on assessing user trust in the SNS itself. Moreover, though studies on users' motives and uses of SNS (e.g., Facebook) have been performed [6,7,8] and some even measured users' privacy perception in different SNS sites [1], few have experimentally identified the relationship among privacy concern, trust, and users' actual behavior. Another vacancy of related works is that few have assessed whether people from different nationalities would possess different privacy concern degrees, and whether their use of SNS would be also different as potentially impacted by their privacy belief and trust in the site.

Thus, in this paper, we report some interesting results from our on-going work in this direction. Specifically, we examined three aspects through the comparison of two countries' SNS users (56 French and 58 Chinese): their privacy concern, overall trust, and actual usage behavior. The reason of selecting the two countries is because they can respectively represent western and oriental cultures, so the discovered differences (if any) could in some sense reflect the two cultures' specialties. Indeed, according to Hofstede's cultural dimensions [4], western countries commonly have individualism and low context culture, whereas eastern countries have collectivism and high context culture. This hence suggests that users from eastern countries would be more addicted to use SNS because it creates a collaborative environment for them to enhance the social affiliation. If so, they would inherently have lower privacy concern and higher trust, in comparison to users from western countries. In the following, we will present experiment setup and results analysis with the objective of verifying this hypothesis.

\section{Experiment Procedure and Instruments}

The survey was launched in Dec. 2010 in the form of online questionnaire. It was announced through public channels in both China and France sides. In the survey, the subject was first asked to fill in her/his demographical information (including age, gender, profession, etc.). S/he was also asked to give one specific SNS that $\mathrm{s} /$ he has used most frequently in the past one year. Then, a set of questions was required to respond, which are mainly about their privacy concern when using internet and SNS, overall trust in SNS, privacy setting, types of personal info that they include in the profile, and whom (e.g., friends, family members, or anyone) they allow to access the profile. Another set of questions was requesting their actual uses, like usage period, daily usage frequency, amount of contacts, the information they have often posted, and so on. Some questions were multi-choices and some were 5-point Likert scale. The concrete questions are listed from Tables 1 to 6 .

Till the end of Jan. 2011, 166 persons volunteered to fill in the questionnaire. We removed users who gave incomplete answers, ones whose indicated sites are not SNS, and ones who used SNS infrequently. Finally, we have 114 active SNS users for the analysis (active users mean that they have used SNS for minimal half of a year and used it on average 1 hour per day).

Among the 58 Chinese users, $39.66 \%$ are males and $60.34 \%$ are females. The average age is 22.9 (ranging from 18 to 49 ). Almost half of users are students in the university and the others work as engineer, researcher, etc. As for the 56 French users, the average age is 27.43 (ranging from 18 to 46 ), with $42.86 \%$ males and $57.14 \%$ females. Their professions include student, engineer, teacher, and officer. Regarding 
the sites that subjects have used most frequently in the past one year, all French subjects stated Facebook. Among Chinese users, RenRen.com was reported by most users (72.4\%), followed by Facebook (17.2\%), Kaixin001.com (3.4\%) and other local variants. Implied from [5], these Chinese sites can be fairly comparable to Facebook, because they share the structural and functional similarities.

\section{Results}

\subsection{Privacy Concern and Trust}

To compare the answers from the two groups of subjects, we used the multivariate analysis of covariance (MANCOVA) because it can reveal whether the differences (if any) can be attributed to the nationality or others (e.g., gender). From Table 1, we can see that French respondents showed higher general privacy concern when using the Internet $\left(Q 1: M_{\text {French }}=3.81\right.$ vs $\left.M_{\text {Chinese }}=3.48, p=0.072\right)$. The question about their SNS profile privacy setting indicated that they rated significantly higher $\left(Q 2: M_{\text {French }}\right.$ $=3.68$ vs $\left.M_{\text {Chinese }}=2.90, p<.001\right)$. In addition, they felt less comfortable when giving personal information on SNS $\left(Q 3: M_{\text {French }}=2.02\right.$ vs $\left.M_{\text {Chinese }}=2.69, p<.001\right)$, less control in specifying and updating their profiles $\left(Q 4: M_{\text {French }}=2.97\right.$ vs $M_{\text {Chinese }}=3.77$, $p<.001)$, and less agreed that their privacy is protected by the site $\left(Q 5: M_{\text {French }}=2.08\right.$ vs $M_{\text {Chinese }}=2.98, p<.001$ ). These differences are all significant. Regarding users' overall trust in SNS, it also showed that the trust level of French users is significantly lower than the level of Chinese $\left(Q 6: M_{\text {French }}=2.22\right.$ vs $\left.M_{\text {Chinese }}=2.65, p<.01\right)$.

In order to examine whether the cultural contexts significantly differentiate users' privacy and trust degrees, we used the six questions above as dependent variables, and

Table 1. MANCOVA results regarding privacy concern and trust: multivariate tests, adjusted means and standard errors

\begin{tabular}{|c|c|c|c|c|c|}
\hline \multirow[t]{3}{*}{$\begin{array}{l}\text { Gender } \\
\text { Nationality }\end{array}$} & \multicolumn{5}{|c|}{$\begin{array}{l}F \\
2.49^{*} \\
9.87^{\text {*** }}\end{array}$} \\
\hline & \multicolumn{2}{|c|}{ France $(N=56)$} & \multicolumn{2}{|c|}{ China $(N=58)$} & $F$ \\
\hline & $M$ & $S E$ & $M$ & $S E$ & \\
\hline $\begin{array}{l}\text { Q1: How often do you concern about your } \\
\text { privacy while you use the Internet? }\end{array}$ & 3.81 & .14 & 3.48 & .13 & $3.29^{*}$ \\
\hline $\begin{array}{l}\text { Q2: How do you rate the privacy setting of } \\
\text { your profile in SNS? }\end{array}$ & 3.68 & .13 & 2.90 & .13 & $18.14^{* * *}$ \\
\hline $\begin{array}{l}\text { Q3: I feel comfortable giving personal } \\
\text { information on SNS. }\end{array}$ & 2.02 & .13 & 2.69 & .13 & $13.40^{* * *}$ \\
\hline $\begin{array}{l}\text { Q4: I feel having control in specifying and } \\
\text { updating my profile in SNS. }\end{array}$ & 2.97 & .13 & 3.77 & .12 & $21.01^{* * *}$ \\
\hline $\begin{array}{l}\text { Q5: I feel that the privacy of my personal } \\
\text { information is protected by SNS. }\end{array}$ & 2.08 & .12 & 2.98 & .12 & $27.41^{* * *}$ \\
\hline Q6: My overall trust in SNS is high. & 2.22 & .11 & 2.65 & .11 & $8.32^{* *}$ \\
\hline
\end{tabular}

Note: All questions were responded on a 5-point Likert scale: $Q 1$ from "very seldom" to "very often"; Q2 from "not private" to "very private"; $Q 3$ - Q6 from "strongly disagree" to "strongly agree".

* Significant at $p<.1{ }^{* *}$ Significant at $p<.01 ;{ }^{* * *}$ Significant at $p<.001$ (the same notations used in the tables below). 
gender as a covariate in MANCOVA analysis. The result showed that subjects with different nationalities did differ significantly (Wilks' $\Lambda=.64, F=9.87, p<.001$ ), with the effect of gender being controlled (Wilks' $\Lambda=.88, F=2.49, p<.05$ ) (Table 1).

Moreover, Table 2 lists the multiple regression results that expose the causal relationship from privacy constructs (Q3 to Q5) to overall trust in SNS (Q6). For French users, the feeling that their privacy is protected by the site significantly leads to their trust in the site $(\beta=.646, p<.001)$, whereas for Chinese, the comfortableness in giving personal information is a significant factor $(\beta=.488, p<.001)$.

Table 2. Multiple regression analyses for predicting user trust

\begin{tabular}{|c|c|c|c|c|c|c|c|c|}
\hline & Franc & & & & China & & & \\
\hline & $\beta$ & $t$ & $R^{2}$ & $F$ & $\beta$ & $t$ & $R^{2}$ & $F$ \\
\hline $\begin{array}{l}\text { "My overall trust in SNS } \\
\text { is high" }\end{array}$ & & & .52 & $19.1^{\text {**** }}$ & & & .22 & $5.07^{\text {sth }}$ \\
\hline Q3 (feel comfortable) & .157 & 1.46 & & & .488 & $3.82^{* * *}$ & & \\
\hline Q4 (feel having control) & .014 & .14 & & & -.164 & -1.28 & & \\
\hline Q5 (feel privacy protected) & 646 & $6.18^{* * *}$ & & & .018 & .15 & & \\
\hline
\end{tabular}

Note: $Q 3$ to $Q 5$ are referred to Table 1

At the next step, we analyzed the sorts of personal information that they included in their SNS profiles (as reported by them). This analysis revealed several significant differences between French and Chinese users (see Table 3). Specifically, Chinese subjects disclosed significantly more identifying information such as gender, email, phone number, and location. For example, $93.1 \%$ of Chinese subjects include their gender (vs. 62.5\% French), 79.3\% share email (vs. 51.8\% French), 77.6\% indicate location (vs. 46.4\%), 55.2\% give instant messenger account (vs. 17.9\% French), $44.8 \%$ include biography info (vs. 10.7\%), and $81.0 \%$ include education info (vs. $42.9 \%)$. On the other hand, more French users share single or married status than Chinese users (55.4\% vs. $36.2 \%)$. As for other items, such as real name, nationality, self picture, birthday, they are popularly disclosed by both user groups (above 70\%), and some items like home address are rarely included by both (below 10\%) (due to space limit, these results are not listed in Table 3). In addition, Chinese users are more frequent in updating their profiles (i.e., "once or several times a week"), compared to French subjects who have updated profiles averagely "once or several times a month".

For the profile, we also asked the subject whom s/he allows to access it. Significant differences occur on the accessible right by friends, classmates, strangers and anyone (Table 4). In fact, more French subjects (96.4\%) indicated that their profiles can be

Table 3. Personal information included in user profile

\begin{tabular}{|c|c|c|c|c|c|c|c|c|c|}
\hline & & Gender & Email & $\begin{array}{l}\text { Phone } \\
\text { number }\end{array}$ & Location & $\begin{array}{l}\text { Single or } \\
\text { married } \\
\text { status }\end{array}$ & $\begin{array}{l}\text { Messenger } \\
\mathrm{a} / \mathrm{c}\end{array}$ & Biography & Education \\
\hline $\begin{array}{l}\text { French } \\
(N=56)\end{array}$ & Mean & $62.5 \%$ & $51.8 \%$ & $5.4 \%$ & $46.4 \%$ & $55.4 \%$ & $17.9 \%$ & $10.7 \%$ & $42.9 \%$ \\
\hline $\begin{array}{l}\text { Chinese } \\
(N=58) \\
\end{array}$ & Mean & $93.1 \%$ & $79.3 \%$ & $31.0 \%$ & $77.6 \%$ & $36.2 \%$ & $55.2 \%$ & $44.8 \%$ & $81.0 \%$ \\
\hline Sig. & & $.003^{* *}$ & $.001^{* *}$ & $.000^{* * * *}$ & $.000 * * *$ & $.041 *$ & $.000^{* * * *}$ & $.000^{* * * *}$ & $.000^{* * *}$ \\
\hline
\end{tabular}


Table 4. Other users' right of accessing the profile

\begin{tabular}{|c|c|c|c|c|c|c|c|c|}
\hline \multicolumn{9}{|c|}{ Your profile can be accessed by..? } \\
\hline & & Friends & $\begin{array}{l}\text { Friends of } \\
\text { your } \\
\text { friends }\end{array}$ & Colleagues & $\begin{array}{l}\text { Family } \\
\text { members }\end{array}$ & Classmates & Strangers & Anyone \\
\hline $\begin{array}{l}\text { French } \\
(N=56)\end{array}$ & Mean & $96.4 \%$ & $10.7 \%$ & $21.4 \%$ & $48.2 \%$ & $19.6 \%$ & $1.8 \%$ & $3.6 \%$ \\
\hline $\begin{array}{l}\text { Chinese } \\
(N=58)\end{array}$ & Mean & $86.2 \%$ & $3.4 \%$ & $29.3 \%$ & $36.2 \%$ & $62.1 \%$ & $10.3 \%$ & $13.8 \%$ \\
\hline Sig. & & $.05^{*}$ & .135 & .338 & .198 & $.000^{* * *}$ & $.05^{*}$ & $.05^{*}$ \\
\hline
\end{tabular}

accessed by friends, than $86.2 \%$ Chinese users $(p<0.05)$. However, Chinese users are more open to allow classmates $(62.1 \%$ vs. $19.6 \%$ French), strangers (10.3\% vs. $1.8 \%)$ and even anyone $(13.8 \%$ vs. $3.6 \%)$ to have the viewing right.

The above findings hence indicate that, when the users have less privacy concern and more trust in SNS (i.e., Chinese subjects), they are likely to disclose more in their profiles and enable more kinds of other users to access the personal info. We were hence motivated to further investigate whether users' actual usages of SNS were also similarly influenced.

\subsection{Usage and New Relationship Development}

As indicated in Section 2, we only analyzed answers from users who have used SNS for at least half of a year, because they can represent active users of SNS. The actual usage duration of Chinese group is 2.69 years on average (ranging from 1 to 4 years), and it is 2.7 in French group (also ranging from 1 to 4 years). Their overall visiting frequency is around "once or more than once per day" in both groups. As for the hours that they usually spent on SNS per day, $19.6 \%$ of French and 36.2\% of Chinese subjects reported that they have used it for 1 to 2 hours, $12.5 \%$ of French and $13.8 \%$ of Chinese have spent 2 to 5 hours, 3.6\% French have used it for above 5 hours, and $64.3 \%$ French and 50\% Chinese have used it for less than 1 hour.

Table 5. Messages that users usually post to SNS

\begin{tabular}{|c|c|c|c|c|c|c|c|c|}
\hline \multicolumn{9}{|c|}{ What do you usually post? } \\
\hline & & News & Hobbies & $\begin{array}{l}\text { Personal } \\
\text { life }\end{array}$ & $\begin{array}{l}\text { Current } \\
\text { event }\end{array}$ & $\begin{array}{l}\text { Interesting } \\
\text { observation }\end{array}$ & $\begin{array}{l}\text { Thoughts/ } \\
\text { opinions }\end{array}$ & $\begin{array}{l}\text { Forward } \\
\text { others' posts }\end{array}$ \\
\hline $\begin{array}{l}\text { French } \\
(N=56)\end{array}$ & Mean & $39.3 \%$ & $21.4 \%$ & $32.1 \%$ & $28.6 \%$ & $30.4 \%$ & $35.7 \%$ & $16.1 \%$ \\
\hline $\begin{array}{l}\text { Chinese } \\
(N=58)\end{array}$ & Mean & $24.1 \%$ & $41.4 \%$ & $62.1 \%$ & $25.9 \%$ & $58.6 \%$ & $46.6 \%$ & $58.6 \%$ \\
\hline Sig. & & $.084^{*}$ & $.02^{*}$ & $.000^{* * *}$ & .748 & $.002^{* * *}$ & .243 & $.000^{* * * *}$ \\
\hline
\end{tabular}

Despite their similar visiting frequency, the number of contacts in their "friends" list was notably different. French users reported that they have on average 183.2 contacts (SD = 202.0), whereas Chinese have 299.1 contacts ( $\mathrm{SD}=342.4)$. In addition, the messages they have usually posted to SNS are also different (Table 5). Chinese users are more active in posting hobbies (41.4\% against $21.4 \%$ French users), 
personal life (62.1 vs. $32.1 \%)$, interesting observation $(58.6 \%$ vs. $30.4 \%)$, and forwarding others' posts $(58.6 \%$ vs. $16.1 \%)$, while French are more active in posting news (39.3\% vs. $24.1 \%$ Chinese). These differences all reach at significant levels.

In this survey, we also asked users whether they like to use SNS to meet new people, and whether they have contacted new people afterwards through other ways (e.g., telephone, email, instant messenger). In this regard, French rated significantly lower for using SNS to meet new people $\left(M_{\text {French }}=1.73\right.$ vs $M_{\text {Chinese }}=2.71, F=33.32$, $p<.001)$. It is hence not surprising that most French subjects did not communicate with new people outside of the site $(60.7 \%$ against $41.4 \%$ Chinese, $p<0.05$; see Table 6). In comparison, Chinese users are more active in contacting new people off SNS by telephone and instant messenger.

Table 6. Other ways that users communicate with new people after they met her/him in SNS

\begin{tabular}{|c|c|c|c|c|c|c|}
\hline & & Telephone & $\begin{array}{l}\text { Face-to-face } \\
\text { meeting }\end{array}$ & $\begin{array}{l}\text { Instant } \\
\text { messenger }\end{array}$ & Email & $\begin{array}{l}\text { Did not communicate } \\
\text { outside of the site }\end{array}$ \\
\hline $\begin{array}{l}\text { French } \\
(N=56)\end{array}$ & Mean & $5.4 \%$ & $17.9 \%$ & $14.29 \%$ & $19.6 \%$ & $60.7 \%$ \\
\hline $\begin{array}{l}\text { Chinese } \\
(N=58)\end{array}$ & Mean & $15.5 \%$ & $17.2 \%$ & $50.0 \%$ & $31.0 \%$ & $41.4 \%$ \\
\hline Sig. & & $.07^{*}$ & .931 & $.000^{* * * *}$ & .164 & $.038^{*}$ \\
\hline
\end{tabular}

Table 7. Multiple regression analyses for predicting visiting frequency, amount of contacts and willingness to meet new people in SNS

\begin{tabular}{|c|c|c|c|c|c|c|c|c|}
\hline & France & & & & China & & & \\
\hline & $\bar{\beta}$ & $t$ & $R^{2}$ & $\bar{F}$ & $\bar{\beta}$ & $t$ & $R^{2}$ & $\bar{F}$ \\
\hline Visiting frequency & & & .259 & $4.447^{* *}$ & & & .089 & 1.297 \\
\hline Q3 (feel comfortable) & .178 & 1.291 & & & .020 & .130 & & \\
\hline Q4 (feel having control) & .262 & $2.009^{*}$ & & & .285 & $2.004^{*}$ & & \\
\hline Q5 (feel privacy protected) & -.219 & -1.263 & & & .024 & .176 & & \\
\hline Q6 (overall trust) & .392 & $2.242^{*}$ & & & .002 & .011 & & \\
\hline Amount of contacts & & & .112 & 1.615 & & & .013 & .175 \\
\hline Q3 (feel comfortable) & .214 & 1.420 & & & -.057 & -.351 & & \\
\hline Q4 (feel having control) & .142 & .993 & & & .054 & .366 & & \\
\hline Q5 (feel privacy protected) & .006 & .029 & & & .044 & .309 & & \\
\hline Q6 (overall trust) & .074 & .387 & & & 107 & .693 & & \\
\hline $\begin{array}{l}\text { "I like to use SNS to meet } \\
\text { new people" }\end{array}$ & & & .215 & $3.493^{*}$ & & & .208 & 3.489 \\
\hline Q3 (feel comfortable) & .231 & 1.629 & & & .215 & 1.470 & & \\
\hline Q4 (feel having control) & .042 & .311 & & & -.136 & -1.029 & & \\
\hline Q5 (feel privacy protected) & -.151 & -.849 & & & .317 & $2.480^{*}$ & & \\
\hline Q6 (overall trust) & .404 & $2.244^{*}$ & & & .134 & .968 & & \\
\hline
\end{tabular}

Note: $Q 3$ to $Q 6$ are referred to Table 1

As shown in Section 3.1, various privacy factors were indicated to be driving forces for influencing French and Chinese users' trust in SNS. Based on it, we were interested in further elaborating the relationships between privacy/trust and users' actual usage behavior. For this purpose, users' overall visiting frequency, amount of 
contacts in their "friends" list, and willingness to meet new people were taken as dependent variables, to be predicted by privacy concern and trust (see Table 7).

In predicting the visiting frequency, control in specifying \& updating profiles takes significantly positive effect in both groups $(\beta=.262, p=0.05$ in French and $\beta=.285$, $p=0.05$ in Chinese). Moreover, increased overall trust can also result in more visits among French subjects $(\beta=.392, p<.05)$, which however is not significantly valid for Chinese. Regarding the amount of contacts, there is no significant predictor found in both groups. For the willingness to meet new people in SNS, the overall trust was shown to be a significant predictor among French $(\beta=.404, p<0.05)$, whereas for Chinese the feeling that their privacy is protected by SNS is the significant predictor $(\beta=.317, p<0.05)$.

\section{Discussion and Conclusions}

Thus, through this user survey, it is interesting to find that French and Chinese SNS users did possess significantly different privacy belief and trust. Specifically, French users are more concerned about their privacy while using the Internet. It hence seems being a natural consequence that their privacy setting in SNS is higher, they felt less comfortable in giving personal info, and they perceived the site less trustworthy to protect their privacy. Their overall trust in SNS is also significantly lower relative to Chinese users'. Such differences were further reflected in their profile disclosure and sharing. Chinese subjects disclosed more kinds of personal info in their profiles, such as gender, phone number, location, biography, education, etc., and allowed different types of users (e.g., including strangers) to access the profile. On the contrary, French users seem conservative in disclosing themselves, and they mainly allow friends and family members to view their profiles.

We further measured users' actual usage patterns. It showed that although both groups exhibit similar usage period and frequency, the ways they post messages and develop new relationships are significantly different. First of all, Chinese users have more contacts in their "friends" list. Secondly, they are more active in posting their hobbies, personal life, interesting observations, and forwarding others' posts, while French users are more active in posting news. Thirdly, Chinese users are more willing to meet new people through SNS and reported that they have contacted new people through other communication ways like telephone and instant messenger. In comparison, French users still behave conservative and most of them indicated that they did not communicate with new people outside of SNS.

The multivariate analysis of covariance (MANCOVA) further verifies that these phenomena can be attributed to the nationality difference. In addition, via the multiple regression analysis, we clarified the causal relation among these three factors: privacy concern, trust and users' actual uses. The analysis between privacy concern and trust first showed that for French users, the extent that they feel the site can protect their privacy is significantly correlated to whether they trust it, whereas for Chinese users, the significantly leading factor is whether they feel comfortable in providing personal info. Furthermore, some privacy factors and trust are found to significantly affect users' usage behavior. For instance, increased control in specifying and updating profiles can prompt both groups of users to visit SNS more often. Moreover, for 
French users, increased trust also results in more visits and higher likelihood to meet new people. For Chinese, the perception with SNS's ability in protecting their privacy will likely result in their willingness to develop new relationships.

Thus, though the subjects' scale is limited in this survey, it revealed significant differences between Chinese and French SNS users from various aspects. We believe that the reason behind can be connected to how an individual internalizes the norms, rules and values in a society, which is in turn shaped by her/his cultural background. French, in a typical Western culture [4], are characterized by higher uncertainty avoidance, self-reliance, and emotional distance from in-groups. It might explain why they care more about their own privacy and attempt to keep distance from others (especially from new people). On the contrary, Chinese, as shaped by collectivistic culture which emphasizes family integrity, in-group membership and interdependence among people, should be in nature more willing to establish close social connections with others and hence be likely to disclose most about themselves. On the other hand, the country's privacy regulation may also influence users' privacy concern. That is, users from countries with omnibus privacy regulatory structure (e.g., France) would have higher levels of privacy concerns, than users from countries with sectoral or no privacy regulation (e.g., China) [9]. In the future, we will conduct more experiments to verify these explanations, and derive design guidelines for SNS so that it could better fulfill the expectations of their users with different cultural norms.

Acknowledgement. We thank Dr. Sylvain Castagnos and Prof. Anne Boyer from LORIA - Nancy University, France, for their help of recruiting French subjects. We are grateful to all survey participants for their time.

\section{References}

1. Dwyer, C., Hiltz, S.R., Passerini, K.: Trust and Privacy Concern Within Social Networking Sites: A Comparison of Facebook and MySpace. In: 13th Americas Conference on Information Systems (2007)

2. Golbeck, J.: Trust and Nuanced Profile Similarity in Online Social Networks. ACM Trans. Web 3(4) (2009)

3. Grabner-Kräuter, S., Kaluscha, E.A.: Empirical Research in On-line Trust: a Review and Critical Assessment. International Journal of Human-Computer Studies 58 (2003)

4. Hofstede, G.: Culture's Consequences: Comparing Values, Behaviors, Institutions, and Organizations across Nations. Sage Publications, Thousand Oaks (2001)

5. Jiang, J., Wilson, C., Wang, X., Huang, P., Sha, W., Dai, Y., Zhao, B.Y.: Understanding Latent Interactions in Online Social Networks. In: Annual Conference on Internet Measurement, pp. 369-382 (2010)

6. Joinson, A.N.: Looking At, looking Up or Keeping Up with People?: Motives and Use of Facebook. In: ACM SIGCHI 2008, pp. 1027-1036 (2008)

7. Kim, Y., Sohn, D., Choi, S.M.: Cultural Difference in Motivations for Using Social Network Sites: A Comparative Study of American and Korean College Students. Computers in Human Behavior 27, 365-372 (2011)

8. Lampe, C., Ellison, N.B., Steinfield, C.: Changes in Use and Perception of Facebook. In: ACM Conference on Computer Supported Cooperative Work, pp. 721-730 (2008)

9. Privacy International Organization, https : / /www. privacyinternational . org 